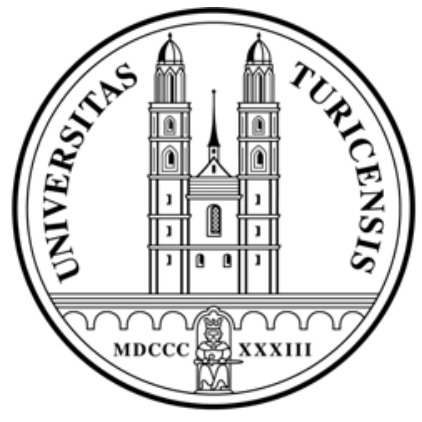

Institute for Empirical Research in Economics

University of Zurich

Working Paper Series

ISSN 1424-0459

Working Paper No. 490

Rent-seeking contests with independent private values

Christian Ewerhart

June 2010 


\title{
Rent-seeking contests with independent private values
}

\author{
Christian Ewerhart ${ }^{a}$
}

${ }^{a}$ University of Zurich; postal address: Chair for Information Economics and Contract Theory, Winterthurerstrasse 30, 8006 Zurich, Switzerland; e-mail: christian.ewerhart@uzh.ch; phone:41-44-6343733; fax: 41-44-6344978.

\begin{abstract}
We consider symmetric rent-seeking contests with independent private valuations of the contest prize. For a two-parameter specification with continuous types, we fully characterize the Bayesian equilibrium, and study its basic properties. The willingness to waste is a hump-shaped function of the private valuation, with the median type expending the highest share of her valuation. A first-order (second-order) stochastic increase in the common type distribution raises (lowers) ex-ante expected efforts. However, neither first-order nor second-order stochastic dominance in valuations necessarily leads to a first-order stochastic dominance ranking in efforts. We also show that, as uncertainty vanishes, the Bayesian equilibrium converges to the Nash equilibrium of the model with complete information.
\end{abstract}

Keywords. Rent seeking; conflict; independent private valuations; firstorder stochastic dominance; mean-preserving spread.

JEL-Codes. C72, H41. 


\section{Introduction}

In a classic rent-seeking contest, as proposed by Tullock (1980), agents expend resources to improve their relative position vis-à-vis others agents with conflicting interests. Such contests may, for instance, relate to lobbying, political campaign, marketing, defense, or sports. In reality, incomplete information, for instance about the valuation of prizes, is a crucial feature of such contests. However, early modeling has largely abstracted from this feature. Therefore, there is a growing interest to understand the implications of asymmetric information for equilibrium behavior in rent-seeking contests.

A number of recent contributions have incorporated private information into the basic set-up. Hurley and Shogren (1998a) consider a model with one-sided private information. I.e., one agent is uncertain about the other agent's valuation of the contest prize. In this case, the uninformed agent's effort becomes a risky input, which reduces her expected probability of winning. Hurley and Shogren (1998b) allow for two-sided private information and correlation. One agent has one of two possible values, the other agent has one of three possible values. In general, this specification allows a numerical solution only. The authors conclude that information is a key factor influencing effort expended in a rent-seeking contest. Restricting attention to a symmetric information structure, with two equally likely values for each agent, Malueg and Yates (2004) fully characterize the Bayesian equilibrium, and show that increased possibilities for ex-post lopsidedness lead to less aggressive bidding. Fey (2008) considers a model with a uniform distribution of marginal costs, and proves existence of a pure strategy Nash equilibrium 
using Schauder's fixed-point theorem. Little is known about the case when players' valuations are drawn from different distributions (cf. Wärneryd, 2010).

In this paper, we consider symmetric rent-seeking contests in which agents have private and independently drawn valuations of the contest prize. Studied is a Bayesian equilibrium, so that an agent's optimal level of expenses depends on her valuation of the contest prize as well as on the equilibrium distribution of effort levels chosen by the other agent. For continuous type distributions, this problem is in general intractable. We will point out, however, that for a particular two-parameter specification with continuous types, the equilibrium indeed allows a closed-form solution. Our main result fully characterizes the symmetric Bayesian equilibrium for this specification.

The characterization obtained can be used to study various properties and comparative statics of the Bayesian equilibrium. Three types of results are derived. First, we show that the willingness to waste, i.e., the share of the prize that an agent is expending for rent-seeking activities, is a hump-shaped function of the agent's valuation. Second, we study the consequences of changing the distribution of types. We find here that a first-order stochastic shift in the common type distribution unambiguously increases ex-ante expected efforts, while a mean-preserving spread in valuations unambiguously lowers ex-ante expected efforts. However, a first-order stochastic increase in types does not necessarily lead to a first-order stochastic increase in equilibrium expenses. Moreover, a second-order stochastic increase in types (i.e., a mean-preserving spread) never leads to a first-order stochastic decrease of bids in our specification. Finally, we show that when the type interval 
converges to a given valuation, the support of the equilibrium strategies converges to the Nash equilibrium of the complete information game.

The rest of the paper is structured as follows. The model is introduced in Section 2. Section 3 presents the two-parameter specification with continuous types, and solves for the equilibrium. Properties and comparative statics of the Bayesian equilibrium are studied in Section 4. Concluding remarks can be found in Section 5. Appendix A replicates our comparative statics results in the two-type specification. The proof of our main result can be found in Appendix B.

\section{Contests with independent private valuations}

Two risk-neutral agents $i=1,2$ compete in a rent-seeking contest that allocates a given prize. Valuations of the prize are random and independently distributed. Denote by $v_{i}$ agent $i$ 's valuation. Valuations are private information, i.e., agent $i=1,2$ observes $v_{i}$, yet not $v_{j}$ for $j \neq i$. The valuation $v_{i}$ is drawn ex ante from a common interval $[\underline{v}, \bar{v}]$, where $0<\underline{v}<\bar{v}$. The density function associated with the distribution of $v_{i}$ is denoted by $f($.$) , the$ corresponding cumulative distribution function by $F($.$) . Agents choose levels$ of expenses simultaneously. We denote by $x_{i} \geq 0$ agent $i$ 's level of expense. The ex-post utility for an agent $i$ with valuation $v_{i}$, for expenses $x_{i}$ by agent $i$, and expenses $x_{j}$ by agent $j \neq i$, is given by

$$
u_{i}\left(x_{i}, x_{j} ; v_{i}\right)=p\left(x_{i}, x_{j}\right) v_{i}-C_{i}\left(x_{i}\right)
$$

where $p\left(x_{i}, x_{j}\right)$ is agent $i$ 's probability of winning (i.e., $p(.,$.$) is the contest$ success function), and $C_{i}\left(x_{i}\right)$ denotes agent $i$ 's costs. We will refer to the 
game of incomplete information thereby defined as a contest with independent private valuations.

Given a contest with independent private valuations, by a symmetric Bayesian equilibrium, we mean a measurable rent-seeking strategy $\xi:[\underline{v}, \bar{v}] \rightarrow$ $\mathbb{R}_{+}$such that for $i=1,2$, and for all $v_{i} \in[\underline{v}, \bar{v}]$, we have

$$
\xi\left(v_{i}\right) \in \arg \max _{x_{i} \geq 0} \int_{\underline{v}}^{\bar{v}} p\left(x_{i}, \xi\left(v_{j}\right)\right) v_{i} f\left(v_{j}\right) d v_{j}-C_{i}\left(x_{i}\right) .
$$

For strictly increasing cost functions one may, as usual, redefine expenses in terms of disutility, and assume without loss of generality that $C_{i}\left(x_{i}\right) \equiv x_{i}$. Moreover, while more general contest success functions can be studied, we will focus in this paper on the lottery success function given by $p\left(x_{i}, x_{j}\right)=$ $x_{i} /\left(x_{i}+x_{j}\right)$ for $x_{i}+x_{j}>0$, and by $p\left(x_{i}, x_{j}\right)=1 / 2$ otherwise. We will impose these two assumptions throughout the paper, and note that the optimization problem in (2) is then well-defined.

While we will restrict attention to contests with private information about valuations, one could equivalently have studied contests with private information about costs (cf. Fey, 2008). Indeed, the equivalence follows directly from dividing the objective function in (2) by $v_{i}>0$. However, for the present purposes, the exposition simplifies somewhat by considering private information about valuations.

\section{A two-parameter specification}

This section deals with a particular specification of a contest with independent private valuations. It has been conjectured by Fey (2008) that the model with uniform cost types is not tractable. However, that conjecture 
leaves open the possibility that a tractable example could be found in other cases. As we will show in this section, the model indeed allows a tractable specification when one is willing to deviate from the uniform case.

To this end, we introduce the following distribution on a given interval $[\underline{v}, \bar{v}]$, for arbitrary parameters $\bar{v}>\underline{v}>0$. For valuations $v \in[\underline{v}, \bar{v}]$, define the function

$$
F(v \mid \underline{v}, \bar{v})=\frac{\ln \left(\sqrt{\frac{(\bar{v}-\underline{v})^{2}}{4}+2(\bar{v}+\underline{v}) v}-\frac{\bar{v}+\underline{v}}{2}\right)-\ln (\underline{v})}{\ln (\bar{v})-\ln (\underline{v})} .
$$

It is immediate to see that $F(. \mid \underline{v}, \bar{v})$ is a cumulative distribution function. Indeed, $F(. \mid \underline{v}, \bar{v})$ is nondecreasing and continuous. Moreover, $F(\bar{v} \mid \underline{v}, \bar{v})=0$ and $F(\bar{v} \mid \underline{v}, \bar{v})=1$. We may therefore assume that the distribution of agents' valuations is given by $F(. \mid \underline{v}, \bar{v})$. As mentioned before, the reason for studying this particular distribution function is that it leads to a tractable description of the Bayesian equilibrium. In the sequel, we often write $F($.$) , dropping the$ reference to the parameters $\underline{v}$ and $\bar{v}$.

Figure 1 below illustrates the associated density $f=F^{\prime}$ for parameters $\underline{v}=8$ and $\bar{v}=24$. 


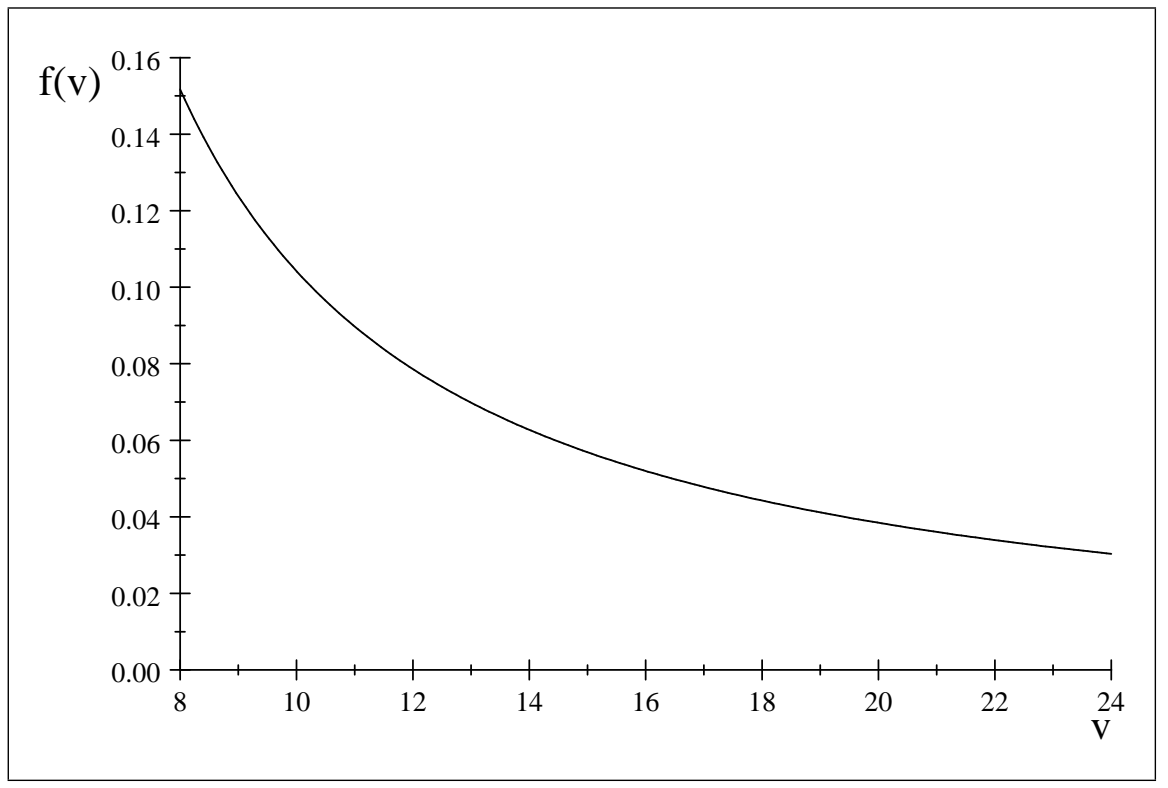

Figure 1. Density of agents' valuations

Having specified the distribution of private valuations, we may now characterize the symmetric Bayesian equilibrium.

Proposition 1. Consider the contest with private valuations drawn independently from the common distribution $F(. \mid \underline{v}, \bar{v})$, where $\bar{v}>\underline{v}>0$. There exists a symmetric Bayesian equilibrium in this contest, in which agents choose the strategy

$$
\xi(v)=\rho\left\{\sqrt{A^{2}+v}-B\right\},
$$

where

$$
\begin{aligned}
A & =\frac{1}{2} \frac{\bar{v}-\underline{v}}{\bar{v}+\underline{v}} \sqrt{\frac{\bar{v}+\underline{v}}{2}}, \\
B & =\frac{1}{2} \sqrt{\frac{\bar{v}+\underline{v}}{2}}, \text { and } \\
\rho & =\frac{1}{\ln (\bar{v} / \underline{v})} \frac{\bar{v}-\underline{v}}{\bar{v}+\underline{v}} \sqrt{\frac{\bar{v}+\underline{v}}{2}} .
\end{aligned}
$$


In this equilibrium, all types expend a strictly positive effort.

A proof of this result can be found in Appendix B.

See Figure 2 for an illustration of the equilibrium strategy.

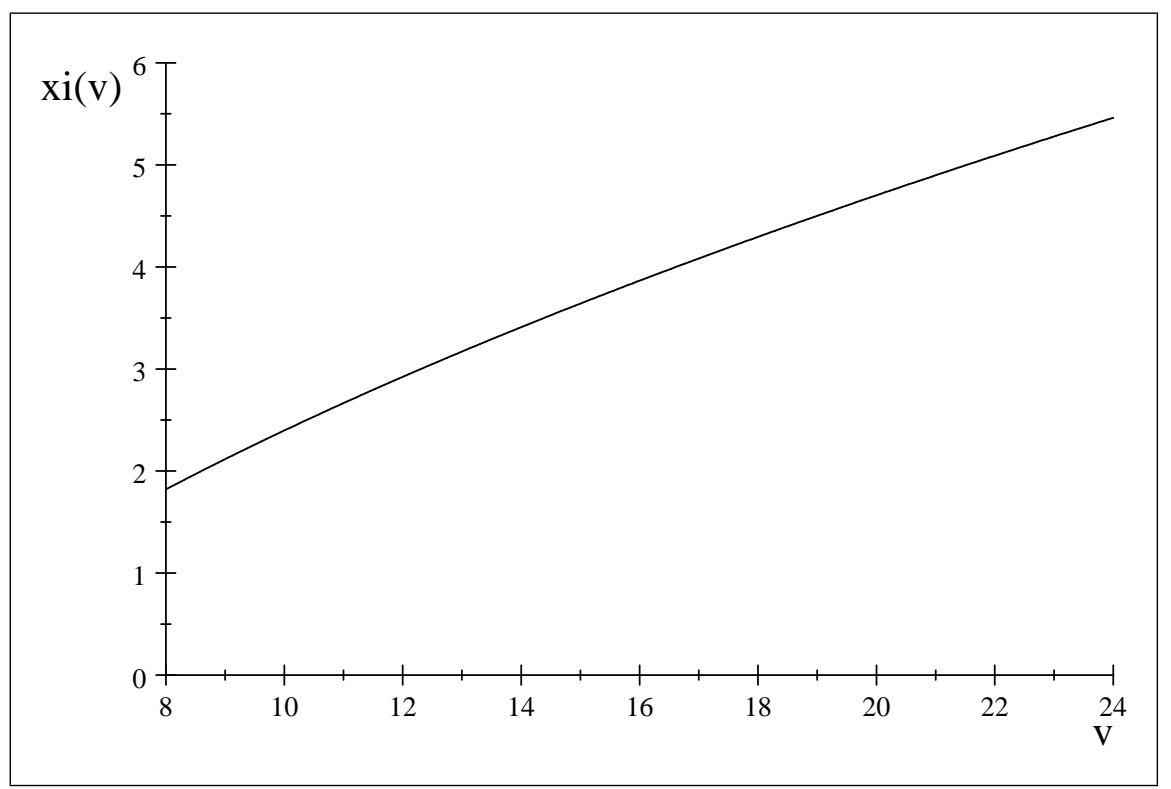

Figure 2. The Bayesian equilibrium strategy

\section{Properties of the equilibrium}

This section derives basic properties and comparative statics of the Bayesian equilibrium identified in Proposition 1. We start by looking at the types' relative willingness to employ resources for inefficient rent-seeking purposes. Subsequently, we will study the consequences of stochastically changing the underlying type distribution. The final subsection contains a limit consideration for vanishing uncertainty. 


\subsection{Equilibrium efforts}

In this subsection, we compare effort levels across types. For this, we will make use of the following concept. Given a symmetric Bayesian equilibrium in a rent-seeking contest, an agent's willingness to waste (Hurley and Shogren, 1998a) is defined as

$$
\beta(v)=\frac{\xi(v)}{v},
$$

where $v$ is the agent's valuation. Thus, $\beta(v)$ is the share of the prize that an agent with valuation $v$ is willing to sacrifice in the contest.

Let us briefly report on what is known about the willingness to waste. Under complete information, the willingness to waste is identical across agents (cf. Hurley and Shogren, 1998b). With two-sided asymmetric information and two equally likely types, the willingness to waste is identical across types as a consequence of the proportionality of equilibrium bids (cf. Malueg and Yates, 2004). With continuous types, the numerical description of the equilibrium strategy (cf. Fey, 2008) suggests that the willingness to waste does not vary very much with the type.

We will show now that in our specification, the willingness to waste is a hump-shaped function of $v$, with the median type being the most wasteful. The first part of this claim follows from differentiating $\beta(v)$ with respect to $v$. Indeed,

$$
\frac{\partial \beta}{\partial v}=\frac{\rho}{2 v^{2} \sqrt{A^{2}+v}}\left(-2 A^{2}-v+2 B \sqrt{A^{2}+v}\right) .
$$

Hence, $\partial \beta / \partial v<0$ if and only if

$$
\frac{\left(2 A^{2}+v\right)^{2}}{A^{2}+v}>4 B^{2} .
$$


After some re-arranging, this inequality reads

$$
\frac{v^{2}}{A^{2}+v}>4\left(B^{2}-A^{2}\right)
$$

Note now that the term on the left-hand side is strictly increasing in $v$. Therefore, the willingness to waste is indeed hump-shaped (cf. Figure 3).

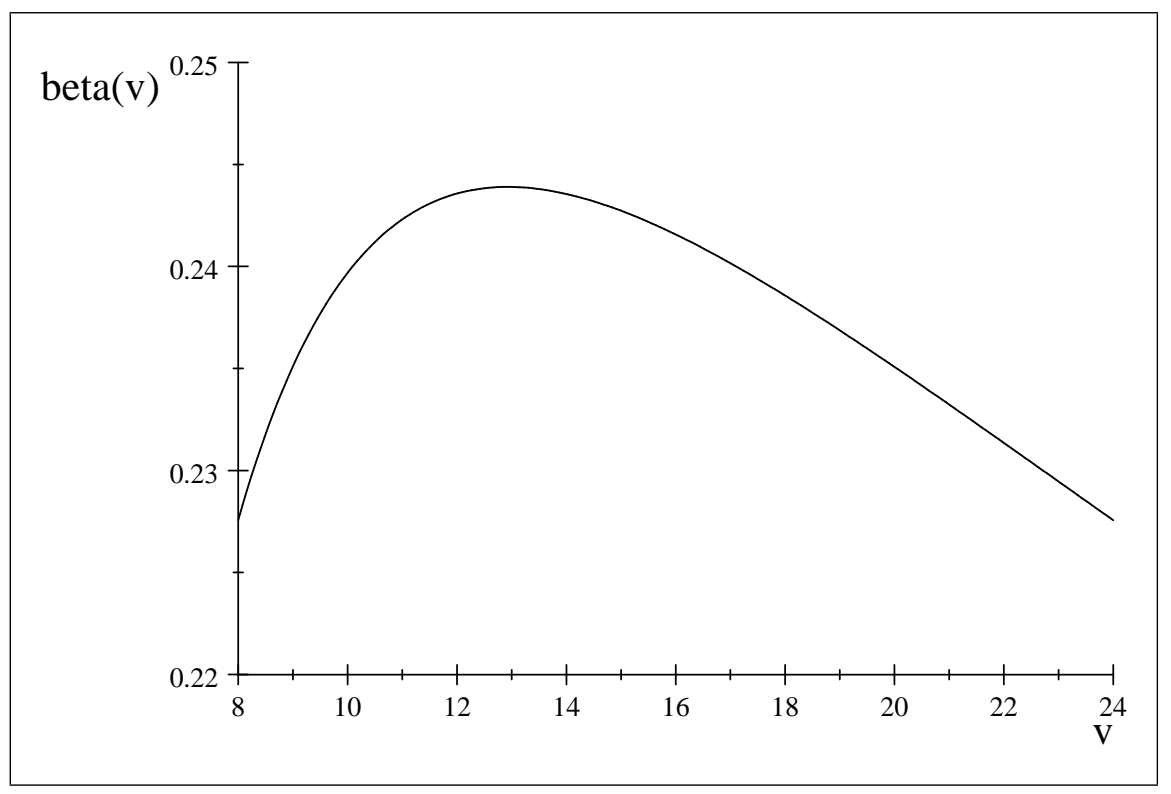

Figure 3. Willingness to waste

As the figure suggests, the willingness to waste for the lowest and the highest valuations is identical. Indeed, using Proposition 1, it is easy to check that

$$
\beta(\underline{v})=\beta(\bar{v})=\frac{1}{2 \ln (\bar{v} / \underline{v})} \frac{\bar{v}-\underline{v}}{\bar{v}+\underline{v}} .
$$

Next we show that the willingness to waste is highest for the median type. The median of the distribution (3) can be found by solving $F(v \mid \underline{v}, \bar{v})=1 / 2$ for $v=v^{M}$. A straightforward calculation shows that the median type is given by

$$
v^{M}=\frac{\underline{v} \bar{v}}{\underline{v}+\bar{v}}+\frac{\sqrt{\underline{v} \bar{v}}}{2} .
$$


For instance, for the parameter values used above, $v^{M}=12.92$. To see that $v^{M}$ indeed maximizes the willingness to waste, one simply checks the first-order condition. As illustrated in Figure 3, the willingness to waste is increasing (decreasing) in the private valuation of the prize for types below (above) the median type.

Intuitively, the hump-shape of $\beta($.$) makes perfect sense. More extreme$ types perceive the contest as more lopsided, which reduces the willingness to waste compared to more central types.

\subsection{Comparative statics}

In this subsection, we use Proposition 1 to study the comparative statics of the symmetric Bayesian equilibrium in the rent-seeking contest. In particular, we are interested to better understand what happens in response to a generally higher level of valuations or in response to a more dispersed type distribution. We have therefore studied (numerically) the consequences of changing the parameters of our specification to reflect a first-order or secondorder stochastic shift in the type distribution. The main steps and results of our extensive calculations are reported upon below. 


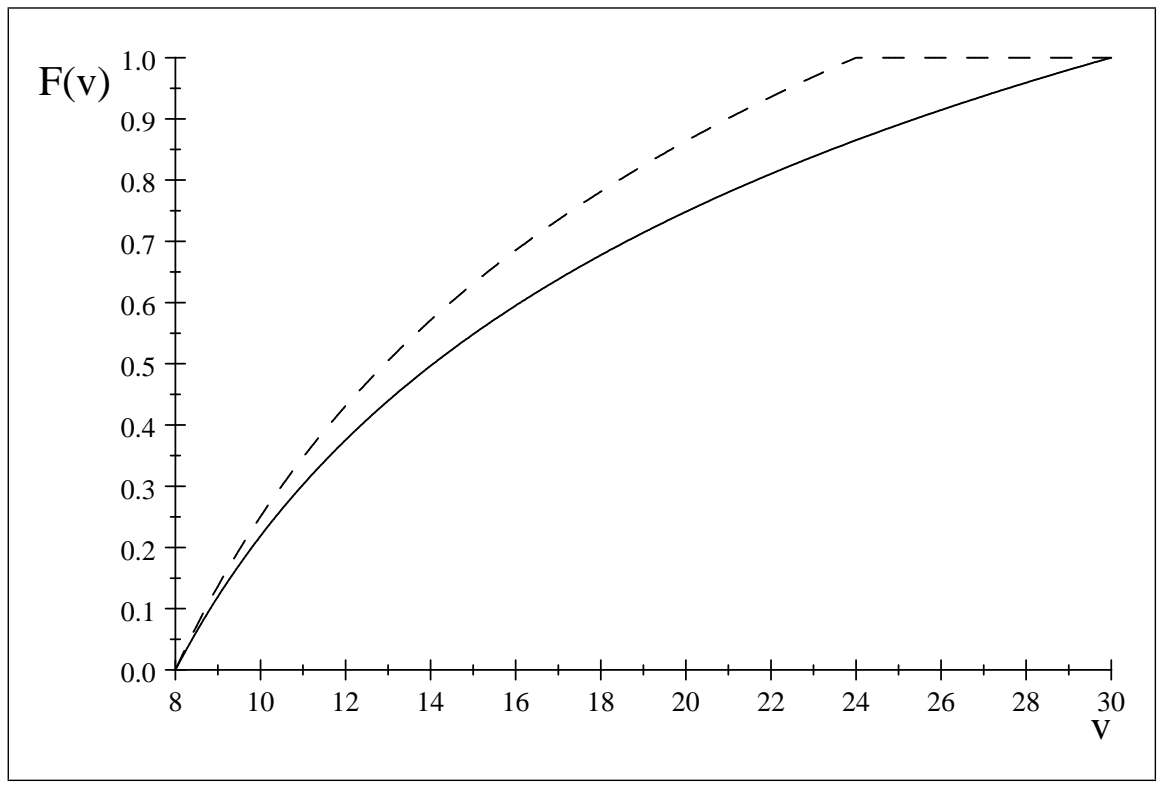

Figure 4. First-order stochastic dominance between type distributions...

First-order stochastic dominance. It is natural to conjecture that increases in either $\bar{v}$ or $\underline{v}$ correspond to first-order stochastic shifts in the type distribution. Numerical tests show that this is indeed the case. We will therefore analyze how the distribution of equilibrium efforts depends on the parameters of our specification.

This requires some preparation. The distribution of equilibrium efforts has the distribution function

$$
G(x \mid \underline{v}, \bar{v}) \equiv F\left(\xi^{-1}(x) \mid \underline{v}, \bar{v}\right)=\frac{\ln \left\{2 x \frac{\bar{v}+\underline{v}}{\bar{v}-\underline{v}} \ln (\bar{v} / \underline{v})\right\}-\ln (\underline{v})}{\ln (\bar{v})-\ln (\underline{v})},
$$

where $\xi^{-1}($.$) denotes the inverse function associated with the strictly increas-$ ing and continuous strategy $\xi($.$) . Instead of studying directly the impact of$ a change of parameters on $G(\cdot \mid \underline{v}, \bar{v})$, it turns out to be easier to consider the $\alpha$-percentile $x=x_{\alpha}(\underline{v}, \bar{v})$ of that distribution, defined as the unique solution 
of the equation $G(x \mid \underline{v}, \bar{v})=\alpha$. A straightforward calculation delivers

$$
x_{\alpha}(\underline{v}, \bar{v})=\frac{\bar{v}^{\alpha} \underline{v}^{1-\alpha}}{2 \ln (\bar{v} / \underline{v})} \frac{\bar{v}-\underline{v}}{\bar{v}+\underline{v}}
$$

which is quite useful for the subsequent analysis.

We may come now to the comparative statics exercises announced above. It turns out that an increase in $\bar{v}$ does not cause bids to increase stochastically. To see this, set $\underline{v}=1$ for convenience. Then

$$
x_{\alpha}(1, \bar{v})=\frac{\bar{v}^{\alpha}}{2 \ln (\bar{v})} \frac{\bar{v}-1}{\bar{v}+1} .
$$

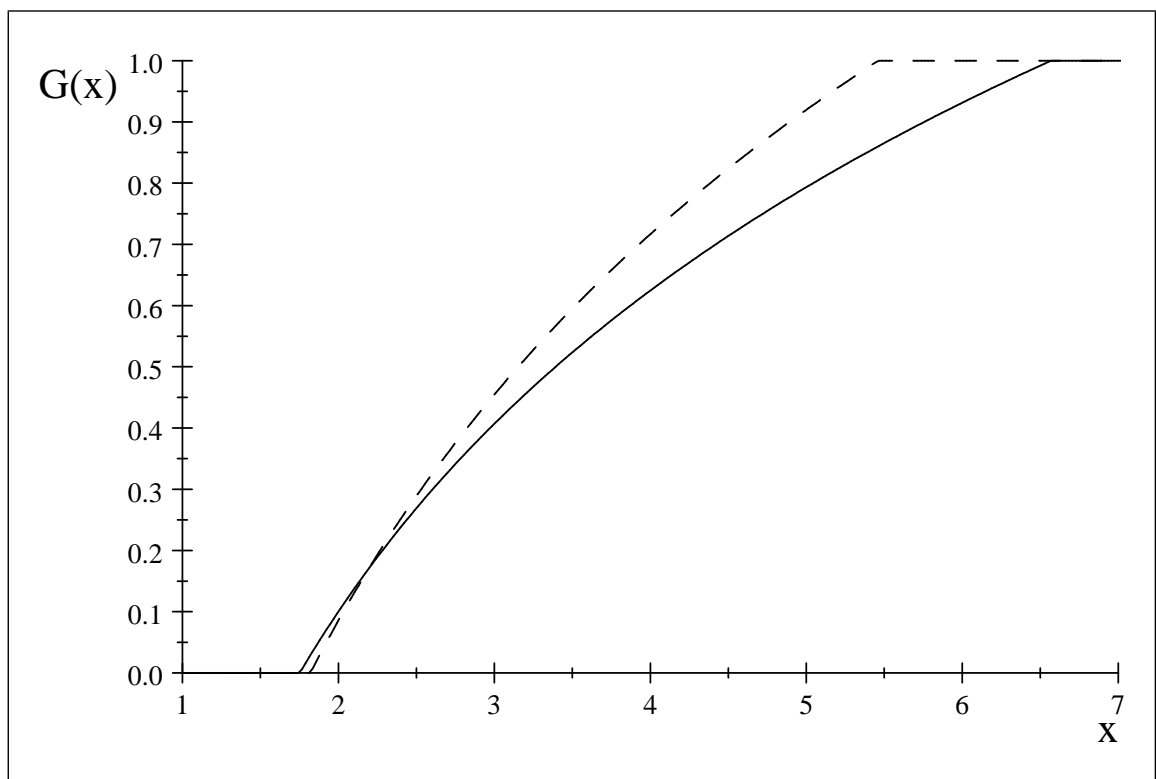

Figure 5. ...does not necessarily imply a first-order stochastic increase of equilibrium efforts.

Numerical calculations show that $x_{\alpha}(1, \bar{v})$ is decreasing in $\bar{v}$ for small $\alpha$, and increasing in $\bar{v}$ for large $\alpha$. See Figure 5 for illustration. This makes sense intuitively because stakes are getting higher for stronger types, while weaker types face even more an uphill battle. The intuitive reason for stochastic 
monotonicity to fail here is therefore that lower types become discouraged through the higher likelihood of meeting a stronger opponent.

An increase in $\underline{v}$, however, provokes a first-order stochastic increase in the distribution of equilibrium efforts. To see this, note that a simultaneous and proportional increase of $\underline{v}$ and $\bar{v}$ by some factor $\lambda>0$ scales up equilibrium strategies by $\lambda$. Therefore, to study comparative statics with respect to $\underline{v}$, we may normalize $\bar{v}$ to one. But

$$
x_{\alpha}(\underline{v}, 1)=-\frac{\underline{v}^{1-\alpha}}{2 \ln (\underline{v})} \frac{1-\underline{v}}{1+\underline{v}} .
$$

Numerical tests verify that this expression is increasing in $\underline{v}$ for any $\alpha \in$ $[0,1]$. Thus, if the lower boundary of the support interval is lifted, all types increase effort. This is intuitive since uncertainty is reduced and all valuations increase.

In sum, we have shown that a first-order stochastic increase in the type distribution may, but need not cause a corresponding first-order stochastic increase in the bid distribution.

Next, we look at the ex ante expected level of expenses of an agent. A straightforward calculation using Proposition 1 delivers

$$
\int_{\underline{v}}^{\bar{v}} \xi(v) f(v) d v=\frac{(\bar{v}-\underline{v})^{2}}{2(\bar{v}+\underline{v}) \ln (\bar{v} / \underline{v})^{2}} .
$$

It turns out that an increase in $\bar{v}$ raises the expected level of expenses. This can be seen through a numerical test after normalizing $\underline{v}=1$, as above. Also an increase in $\underline{v}$ increases the expected level of expenses. Indeed, this follows immediately from the fact that the distribution of equilibrium efforts increases stochastically in $\underline{v}$. Thus, a first-order stochastic increase in the 
type distribution unambiguously raises average efforts in our specification. ${ }^{1}$

Second-order stochastic dominance. It is useful to look at an example. Figure 6 depicts distribution functions $F(. \mid 8,24)$ and $F(. \mid a, 30)$, where the parameter $a=6.23$ has been determined via a numerical integration in such a way that both distributions have the same mean. In addition to the equality of the means, second-order stochastic dominance (cf. Rothschild and Stiglitz, 1970) requires that

$$
\int_{0}^{v_{0}} F(v \mid a, 30) d v \geq \int_{0}^{v_{0}} F(v \mid 8,24) d v
$$

for any $v_{0} \geq 0$. These inequalities have been checked numerically. ${ }^{2}$

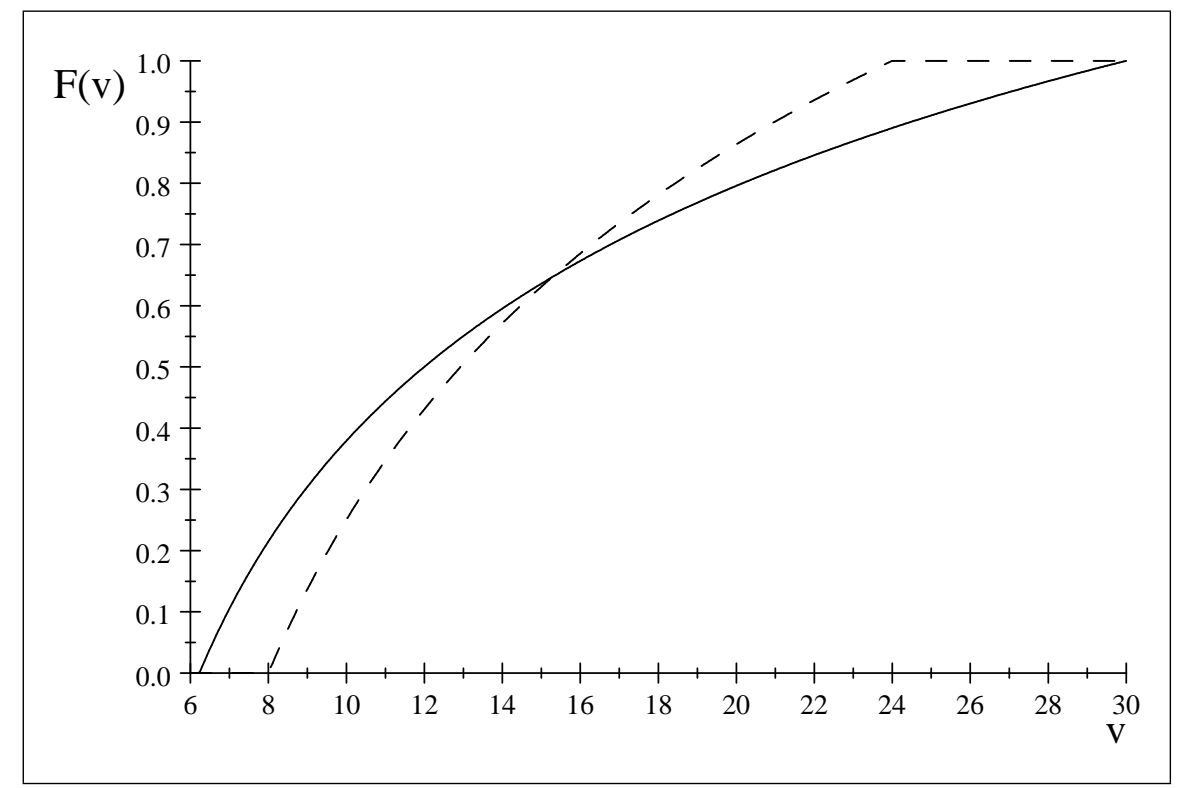

Figure 6. Similarly, second-order stochastic dominance in the type distribution...

\footnotetext{
${ }^{1}$ Maybe interestingly, these predictions for the continuous specification can be replicated in the two-type specification studied, e.g., by Malueg and Yates (2004). I.e., also with two types, a first-order stochastic increase in the type distribution raises average effort, but need not necessarily lead to a first-order stochastic dominance in the distribution of efforts. This is shown formally in Appendix A.

${ }^{2}$ Numerical integrations have been calculated using 1000 grid points in an MS Excel spreadsheet.
} 
We found that expected equilibrium expenses are declining when types are subject to a shift in second-order stochastic dominance.

Figure 7 shows the distribution of equilibrium expenses for our two example distributions. Here, the increase in dispersion of types causes the support interval of equilibrium bids to spread out. In particular, there is no first-order stochastic shift to the level of expenses. Intuitively, this is because a more dispersed type distribution entails additional, aggressive types with very high valuations. ${ }^{3}$

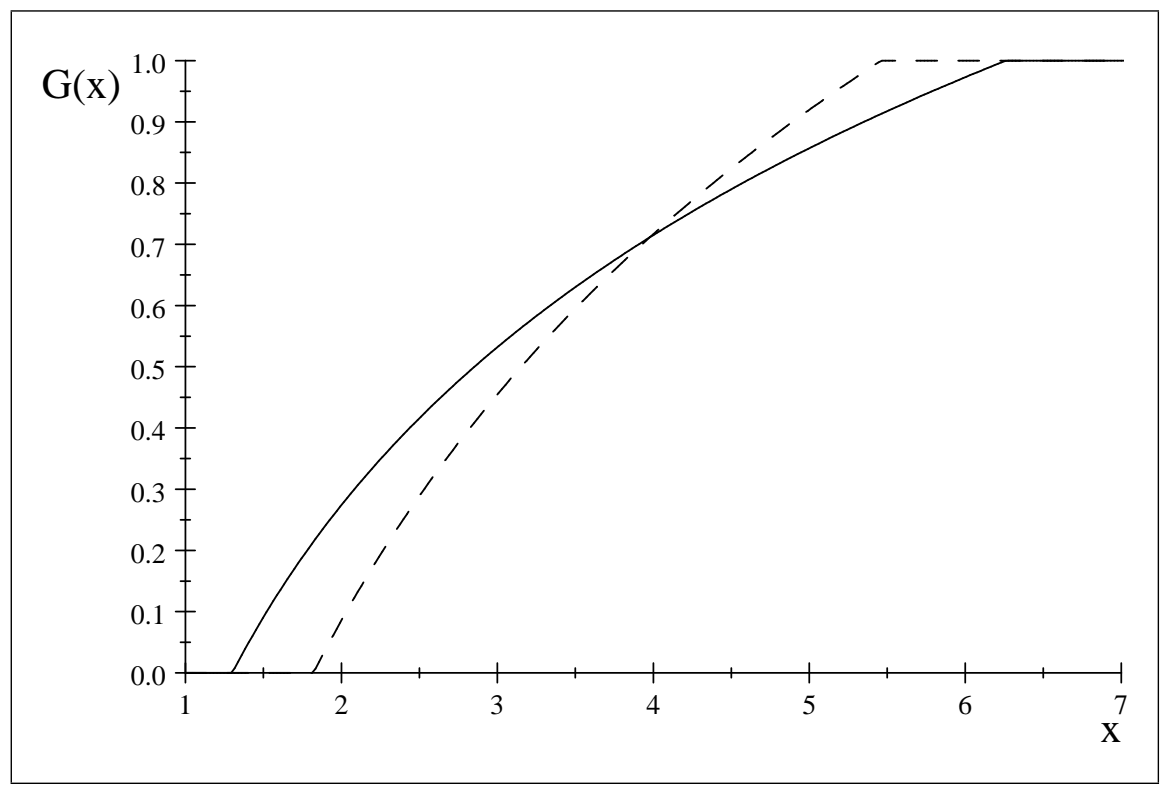

Figure 7. ...does not cause a first-order stochastic lowering in bids.

\subsection{Diminishing uncertainty}

Finally, we remark that the support of the Bayesian equilibrium strategy, as uncertainty vanishes, shrinks down to the equilibrium level of expenses

\footnotetext{
${ }^{3}$ Also the results related to second-order stochastic dominance can be replicated in the two-type specification. See Appendix A.
} 
of the complete-information limit game. To see this reflected in the formal framework, note that as $\bar{v}, \underline{v} \rightarrow V$, we have

$$
\lim _{\substack{\bar{v}, \underline{v} \rightarrow V \\ \bar{v}>\underline{v}}} \frac{\bar{v}-\underline{v}}{\ln (\bar{v})-\ln (\underline{v})}=V .
$$

Hence, from Proposition 1, the limit of

$$
\xi(V)=\rho\left\{\sqrt{A^{2}+V}-B\right\}
$$

is simply $x=V / 4$, which is the Nash equilibrium level of expenses in the symmetric model with complete information. Thus, the specification with complete information can be seen as a limit of continuous specifications with incomplete information.

\section{Conclusion}

The present paper has continued the study of contests with two-sided incomplete information. We have introduced a two-parameter specification of the symmetric lottery contest with continuous types and independent private valuations. Our main result fully characterizes the Bayesian equilibrium for this specification. We have used our result to derive various structural predictions related to the willingness-to-waste, stochastic dominance relationships, and vanishing uncertainty. Of course, there is no guarantee that all of these predictions can be taken for granted in the most general settings. However, one can speculate that some of them, such as the effect of first-order and second-order stochastic dominance on expected expenses, might indeed be valid under more general circumstances. 


\section{Acknowledgements}

My interest in rent-seeking contests was sparked during an interesting conversation with Karl Wärneryd.

\section{Appendix A: Stochastic dominance in the two-type specification}

The purpose of this appendix is it to outline the implications of first-order and second-order stochastic dominance shifts in the two-type specification studied by Malueg and Yates (2004) and others. More specifically, we will show that our comparative statics results for the continuous specification can be replicated in the two-type specification.

For this, consider a symmetric lottery contest with two equally likely, independently drawn type realizations $v_{L}$ and $v_{H}$. Assume $v_{H}>v_{L}>0$. It is well-known that this contest has a unique Bayesian equilibrium in which the willingness to waste is identical across types and equal to

$$
\kappa=\frac{1}{8}+\frac{v_{L} v_{H}}{2\left(v_{L}+v_{H}\right)^{2}}
$$

A first-order stochastic increase of the type distribution corresponds to a simultaneous increase in both $v_{L}$ and $v_{H}$. We claim that a first-order stochastic increase in types causes expected equilibrium effort to increase, whereas a first-order stochastic increase in efforts is not a necessary consequence. To see this, note that expected equilibrium effort is

$$
E[x]=\frac{\kappa}{2}\left(v_{L}+v_{H}\right)=\frac{v_{L}+v_{H}}{16}+\frac{v_{L} v_{H}}{4\left(v_{L}+v_{H}\right)} .
$$

It is not difficult to check that this expression is increasing in both $v_{L}$ and $v_{H}$, proving the first claim. To see that a first-order stochastic increase in efforts 
need not obtain, look at an increase of $v_{H}$ only. The contest becomes then more lopsided, and $\kappa$ decreases. Hence, the equilibrium level of expenses for the low type, $x_{L}=\kappa v_{L}$, is declining.

To investigate the consequence of a second-order stochastic increase of the type distribution, write $\mu=\left(v_{H}+v_{L}\right) / 2$ and $\sigma=\left(v_{H}-v_{l}\right) / 2$. We obtain equilibrium efforts

$$
\begin{aligned}
x_{L} & =\frac{1}{8}\left(2-\frac{\sigma^{2}}{\mu^{2}}\right)(\mu-\sigma) \\
x_{H} & =\frac{1}{8}\left(2-\frac{\sigma^{2}}{\mu^{2}}\right)(\mu+\sigma)
\end{aligned}
$$

Expected equilibrium effort is consequently given by

$$
E[x]=\frac{\mu}{8}\left(2-\frac{\sigma^{2}}{\mu^{2}}\right) .
$$

A mean-preserving spread is now tantamount to increasing $\sigma$. It is therefore immediate from (26) that the expected equilibrium effort is declining when the type distribution undergoes a second-order stochastic dominance shift. We claim that a first-order stochastic decline in bids, however, does not occur as a consequence of a mean-preserving spread in the type distribution. The reason for this to happen is that the high type will always increase her equilibrium effort. To see this formally, note that

$$
\frac{\partial x_{H}}{\partial \sigma}=\frac{1}{4}\left(1-\frac{\sigma}{\mu}\right)>0
$$

\section{Appendix B: Proof of Proposition 1}

This Appendix contains the proof of the equilibrium characterization in the continuous case. We will check the first-order condition for an interior maximum, and subsequently verify that a boundary solution is not feasible. 
Assume that agent $j$ adheres to the candidate equilibrium strategy $\xi($.$) ,$ and that agent $i$ 's type realizes to $v_{i}$. Then the first-order condition corresponding to the agent's optimization problem reads

$$
\int_{\underline{v}}^{\bar{v}} \frac{\xi\left(v_{j}\right) v_{i}}{\left(x_{i}+\xi\left(v_{j}\right)\right)^{2}} d F\left(v_{j} \mid \underline{v}, \bar{v}\right)=1
$$

Using the explicit expression for agent $j$ 's strategy

$$
\xi\left(v_{j}\right)=\rho\left\{\sqrt{A^{2}+v_{j}}-B\right\}
$$

as well as the density of agent $j$ 's value distribution

$$
f\left(v_{j} \mid \underline{v}, \bar{v}\right)=\frac{1}{2 \ln (\bar{v} / \underline{v}) \sqrt{A^{2}+v_{j}}\left\{\sqrt{A^{2}+v_{j}}-B\right\}},
$$

the first-order condition can be re-written as

$$
\int_{\underline{v}}^{\bar{v}} \frac{1}{\left(x_{i}+\rho_{j}\left\{\sqrt{A_{j}^{2}+v_{j}}-B_{j}\right\}\right)^{2}} \frac{\rho_{j} d v_{j}}{2 \sqrt{A_{j}^{2}+v_{j}}}=\frac{\ln (\bar{v} / \underline{v})}{v_{i}} .
$$

The integral is solved by the substitution

$$
Z=x_{i}+\rho_{j}\left\{\sqrt{A_{j}^{2}+v_{j}}-B_{j}\right\}
$$

This yields

$$
\frac{1}{x_{i}+\rho\left\{\sqrt{A^{2}+\underline{v}}-B\right\}}-\frac{1}{x_{i}+\rho\left\{\sqrt{A^{2}+\bar{v}}-B\right\}}=\frac{\ln (\bar{v} / \underline{v})}{v_{i}} .
$$

Using

$$
\begin{aligned}
& \underline{v}=4 B(B-A), \\
& \bar{v}=4 B(B+A),
\end{aligned}
$$

we obtain

$$
\frac{1}{x_{i}+\rho(B-A)}-\frac{1}{x_{i}+\rho(B+A)}=\frac{\ln (\bar{v} / \underline{v})}{v_{i}} \text {. }
$$


Multiplying through, one arrives at a quadratic equation characterizing the optimal level of expenses for agent $i$ :

$$
0=x_{i}^{2}+2 x_{i} \rho B+\rho^{2}\left(B^{2}-A^{2}\right)-\frac{2 A \rho}{\ln (\bar{v} / \underline{v})} v_{i}
$$

Applying the standard formula for quadratic equations yields

$$
x_{i}=-\rho B+\sqrt{\rho^{2} A^{2}+\frac{2 A \rho}{\ln (\bar{v} / \underline{v})} v_{i}},
$$

where one solution drops out due to the nonnegativity requirement on $x_{i}$. Comparing this with the hypothesized equilibrium choice

$$
x_{i}=\rho\left\{-B+\sqrt{A^{2}+v_{i}}\right\}
$$

for agent $i$ shows that it satisfies the first-order condition. It is straightforward to verify that agent $i$ 's problem is strictly concave. Indeed,

$$
(-2) \int_{\underline{v}}^{\bar{v}} \frac{\xi\left(v_{j}\right)}{\left(x_{i}+\xi\left(v_{j}\right)\right)^{3}} v_{i} f\left(v_{j}\right) d v_{j}<0 .
$$

Hence, the solution to the first-order condition is indeed the only solution. This proves the first assertion. To see that all types invest a positive amount for rent-seeking purposes, one plugs the explicit expressions (5) and (6) into (4) to obtain

$$
\xi(\underline{v})=\frac{\rho \underline{v}}{\bar{v}+\underline{v}} \sqrt{\frac{\bar{v}+\underline{v}}{2}}>0 .
$$




\section{References}

Fey, M. (2008). Rent-seeking contests with incomplete information, Public Choice 135: 225-236.

Hurley, T., and Shogren, J. (1998a). Effort levels in a Cournot Nash contest with asymmetric information, Journal of Public Information 69: 195-210.

Hurley, T., and Shogren, J. (1998b). Asymmetric information contests, European Journal of Political Economy 14: 645-665.

Malueg, D., and Yates, A. (2004). Rent seeking with private values, Public Choice 119: 161-178.

Rothschild, M., and Stiglitz, J. (1970). Increasing risk: I. A definition, Journal of Economic Theory 2: 225-243.

Tullock, G. (1980). Efficient rent seeking. In J. Buchanan, R. Tollison \& G. Tullock (eds.), Towards a theory of the rent-seeking society (pp. 97-112). College Station: Texas A \& M University Press.

Wärneryd, K. (2010). Informational Aspects of Conflict, to appear in: Oxford Handbook of the Economics of Peace and Conflict (eds. M. Garfinkel and S. Skaperdas). 